\title{
The attentional control of lexical processing pathways: Reversing the word frequency effect
}

\author{
DAVID A. BALOTA, MARK B. LAW, and JASON D. ZEVIN \\ Washington University, St. Louis, Missouri
}

\begin{abstract}
In two experiments, we investigated the influence of word frequency in speeded word naming and in a relatively novel regularization task in which participants were required to pronounce words on the basis of spelling-to-sound correspondences instead of giving their normal pronunciations (e.g., pronounce pint so that it rhymes with hint). Participants were presented high- and low-frequency regular words and exception words, along with a set of nonwords. The results indicated that there was a normal word frequency effect (i.e., high-frequency words faster than low-frequency words) in the standard speeded naming task, whereas, for the regularization task, the word frequency effect was reversed for regular words, even though the regular words were pronounced in an identical fashion in both the normal naming and the regularization tasks. This reversal of the word frequency effect was not obtained for the exception words. The discussion focuses on the implication of these results for attentional control models of lexical processing.
\end{abstract}

One of the more intriguing aspects of processing visually presented words is that each word affords a number of relatively distinct processing dimensions. Although in most natural language contexts, attention is primarily directed to the meaning of words and their relations to other words, it is quite possible to switch attention to alternative processing dimensions, such as basic visual features (e.g., identifying the color of the script), orthography (e.g., counting the number of consonants), phonology (e.g., determining whether a given word rhymes with another word), and syntax (e.g., identifying the grammatical class). Of course, the extent to which one can switch attention among these processing dimensions depends in large part on the strength of the competing dimensions. Such competition is nicely exemplified in the Stroop color-naming task, wherein there is competition between the strong word dimension and the weaker color dimension, leading to considerable disruption when the two codes are in conflict (Stroop, 1935).

Most models of word processing have emphasized the relatively static character of the lexical processing architecture. For example, in the classic logogen model developed by Morton (1969), the emphasis was on the passive accumulation of featural information within frequencymodulated word recognition devices called logogens. Once the logogen's threshold was surpassed, the word was "recognized." Although there have been changes in the architecture of more recent models, the emphasis on a relatively passive accumulator of information has been

This research was supported by NIA Grant AGO3991. We thank Sally Andrews, Mike Cortese, Steven Lupker, Bob Lorch, David Plaut, and Jason Watson for helpful comments on an earlier version of this manuscript. Correspondence concerning this article should be addressed to D. A. Balota, Department of Psychology, Washington University, St. Louis, MO 63130 (e-mail: dbalota@artsci.wustl.edu). incorporated within the McClelland and Rumelhart (1981) interactive activation model, the Seidenberg and McClelland (1989) parallel distributed processing model, and its more recent descendent, the Plaut, McClelland, Seidenberg, and Patterson (1996) model. In fact, one of the guiding principles of word recognition research appears to be the identification of characteristics that are beyond the attentional control of the individual and, hence, reflect architectural design features of the lexical processing system (see, e.g., Lukatela \& Turvey, 1994; Neely, 1977; Swinney, 1979). This is precisely the type of lexical processing system that was highlighted in arguments concerning modularity (Fodor, 1983).

An alternative approach to lexical processing is to consider the fact that there are multiple pathways engaged when a word is presented and that the influence of each pathway on task performance is dependent on (1) the relative strength of each pathway, (2) the relevance of each pathway for accomplishing the goals of the task, and (3) the integrity of the attentional control system (see Balota, Paul, \& Spieler, 1999). In order to explore this framework, we will attempt to change the relevance of different lexical pathways for accomplishing the goals of a relatively novel task ${ }^{1}$ (pronouncing a word on the basis of common spelling-to-sound principles-e.g., pint so that it rhymes with hint). We will investigate the ramifications of this novel task on the most robust and well-studied effect in the lexical processing literature, the word frequency effect.

Our departure in examining the word frequency effect capitalizes on the assumption that there are distinct roles for whole-word frequency on the two processing pathways that have been proposed by dual-route accounts of speeded word naming (e.g., Coltheart, 1978; Coltheart, Curtis, Atkins, \& Haller, 1993; Monsell, Patterson, Graham, Hughes, \& Milroy, 1992; Paap \& Noel, 1991). According to this framework, one pathway (the lexical route) 
maps the orthographic pattern onto a whole-word representation (much in the spirit of Morton's logogen model) and then simply accesses the pronunciation code that is available from that lexical representation. The second pathway (the sublexical route) captures the spelling-to-sound principles of the language. According to the dual-route framework, word frequency primarily modulates the lexical pathway, because frequency is defined as the frequency of the whole lexical form. In fact, irregular spelling-tosound correspondences often occur in high-frequency words, such as in the irregular pronunciation of ave in the high-frequency word have. Thus, frequency of the wholeword pattern is not a strong predictor of the frequency of the sublexical spelling-to-sound correspondence.

Although the dual-route framework has been useful in accounting for a number of interesting phenomena, including aspects of acquired and developmental dyslexia (e.g., Marshall \& Newcombe, 1980; Shallice \& Warrington, 1980) and cross-linguistic studies of orthographies at varying degrees of spelling-to-sound regularity (e.g., Frost, Katz, \& Bentin, 1987), we presently focus on how the framework accounts for the frequency $\times$ regularity interaction (see, e.g., Andrews, 1982; Monsell et al., 1992; Seidenberg, Waters, Barnes, \& Tanenhaus, 1984; Taraban \& McClelland, 1987). This interaction reflects the finding that there is often a larger influence of regularity for low-frequency words ( pint vs. fern) than for highfrequency words (have vs. then). Although there are clearly alternative accounts of this interaction (e.g., Plaut et al., 1996; Seidenberg \& McClelland, 1989), which we will discuss below, the dual-route framework accommodates this interaction by appealing to differences between the roles of whole-word frequency for the lexical and sublexical routes. Because the lexical route is frequency modulated, dual-route theorists argue that there is little influence of irregular sublexical computations for high-frequency words (e.g., have). The notion is that highfrequency words are processed so quickly (or produce a stronger signal, as in Coltheart et al., 1993) via the lexical route that the computations are completed before there is competition from the sublexical route. On the other hand, for an irregular low-frequency word (e.g., pint), the output from the lexical route is relatively slow, and hence the computations from the lexical route compete for output with the computations from the sublexical route (e.g., pronouncing pint so that it rhymes with hint). In this way, the dual-route framework accommodates the larger regularity effect for low-frequency words than for high-frequency words.

In most word-naming experiments, the primary task directs attention to the lexical processing pathway - that is, participants are told to name "words." In the present study, we will address the ramifications of directing attention to the sublexical pathway. Interestingly, there have already been a number of attempts to do this with English orthography (e.g., Andrews, 1982; Frederiksen \& Kroll, 1976; Midgley-West, 1979; Monsell et al., 1992). ${ }^{2}$
For example, Monsell et al. (1992) manipulated the presence of low-frequency exception words or nonwords within a list in order to direct attention to the lexical and sublexical pathways, respectively. Although Monsell et al.'s (1992) initial results were supportive of attentional control of lexical and sublexical pathways in naming, recent evidence by Lupker, Brown, and Colombo (1997) and Jared (1997) has suggested that changes in response deadlines owing to overall list context is more likely to provide an account of the pattern observed by Monsell et al. (1992; see, however, Zevin \& Balota, 2000, for further evidence from a priming paradigm that one can modulate attention differentially to the lexical and sublexical pathways).

Balota and Ferraro (1996) attempted to investigate the role of attention in lexical and sublexical processes by varying task demands. In this study, participants made rhyme decisions to both words and nonwords. The nonwords were included to encourage attention to the sublexical pathway. Balota and Ferraro suggested that rhyme decisions, along with the presence of nonwords, should increase the importance of the spelling-to-sound correspondence and, hence, discourage the reliance on lexical processing. Interestingly, the results indicated that the word frequency effect was totally eliminated in the rhyming task for a set of stimuli that produced quite large frequency effects in a lexical decision task. This pattern was viewed as supporting the notion that participants attended more to the sublexical pathway for making rhyme decisions than for making lexical decisions.

In the present study, we took a relatively extreme approach to the direction of attention to lexical and sublexical processing information. Specifically, half of the participants were required to directly engage sublexical information -that is, to pronounce letter strings on the basis of the most common spelling-to-sound principles (e.g., pint should be pronounced such that it rhymes with hint). The remaining participants were required simply to name the stimuli correctly. In this way, we were able to compare normal naming, in which lexical information should dominate, to regularization performance, in which sublexical information should dominate. Nonwords were also included in order to further establish the utility of directing attention to the sublexical route, as in the Monsell et al. (1992) and Balota and Ferraro (1996) experiments discussed above. In addition to the nonwords, we included a factorial crossing of spelling-to-sound consistency and word frequency. The presence of the exception words was quite important in ensuring that the participants would attempt to attend to the sublexical pronunciation, because these items would produce a different regularization response than that produced by the more commonly used lexical pathway.

The predictions for the regular words are the primary focus here because, in contrast to the exception words, the actual pronunciation of these items should be identical in both the regularization and the normal naming instructions. Of course, one would expect a word frequency effect 
for the regular words under normal naming instructions. More important, however, is the prediction regarding the relative size of the frequency effect as a function of regularization versus normal naming instructions. If under the regularization instructions, participants can fully rely on a frequency-independent sublexical route, one would expect an elimination of the word frequency effect for these items. If the lexical processing system is more static and immune to attentional control, one might expect similar effects of frequency in both the normal naming and the regularization conditions. As we shall see below, however, the relative difficulty of engaging sublexical processes in the regularization task constrains these simple predictions.

In order to maximize efficiency in reporting the present results, we will report the results from two experiments as a single study and will include experiment as a factor in the analyses. The design of the experiments was identical, with the primary difference between the two experiments being the inclusion of a different set of regular words.

\section{METHOD}

\section{Participants}

In Experiment 1, 20 individuals participated in the normal naming task, and 21 participated in the regularization task, whereas in Experiment 2, 30 individuals participated in the normal naming task and 31 participated in the regularization task. All the individuals were recruited from Washington University undergraduate and graduate courses. The students were paid $\$ 5$ for their participation.

\section{Apparatus}

In Experiment 1, an Apple IIe computer was used to control the displays of the stimuli and to collect response latencies to the nearest millisecond. The stimuli were displayed in white on a black background. In Experiment 2, a 386 PC was used to control the experiment, and the stimuli were displayed on a NEC4G 14-in. color VGA monitor in 80-column mode in white on a black background. In both experiments, response latencies were measured with a Gerbrands Model G1341T voice-operated relay that was interfaced to the computers.

\section{Stimuli}

A total of 120 target stimuli were used in Experiment 1, of which 80 were words ( 20 in each of four categories that were produced by crossing frequency with regularity) and 40 were pronounceable nonwords. For simplicity, we are defining an irregular word as an item that has an aiternative and a common spelling-to-sound mapping at the level of graphemes or word bodies. Two words in the lowfrequency exception word condition were eliminated because of geographical differences in pronunciation, thereby leaving 18 words in this cell.
Experiment 2 also included a total of 120 target words, of which 80 were words ( 20 in each of the four categories that were produced by crossing frequency with regularity) and 40 pronounceable nonwords. There were 20 new low-frequency regular words, and because of a coding error, there were only 19 new high-frequency regular words. The word dark being the only regular word used in both experiments. There were 24 exception words that overlapped with the 40 exception words used in Experiment 1.

The median frequency per million (Kučera \& Francis, 1967, norms), mean length in letters, and mean characteristics of the orthographic neighborhoods for test stimuli are presented in Table 1. An orthographic neighbor was defined as a word that could be produced by changing one letter in the target stimulus. The sum of the neighbors is listed as Coltheart's $N$ in Table 1 (Coltheart, Davelaar, Jonasson, \& Besner, 1977). An orthographic friend was defined as a neighbor that yielded a rhyme of the target (e.g., lint for hint), whereas an orthographic enemy was defined as an orthographic neighbor that yielded a different pronunciation (e.g., hint is an orthographic enemy of pint). As is shown, the high- and low-frequency words were well equated on length and on total number of orthographic friends and enemies. As is shown in Table 1, the regular words did not have zero enemies. This was due to a few items with relatively uncommon spelling-to-sound patterns (e.g., bass for glass). Eliminating such outliers did not change the results of either experiment. Finally, the 40 nonwords were produced by randomly selecting 10 words from each of the four word conditions and changing their letters to produce pronounceable nonwords. The critical stimuli and the matched nonwords used in both Experiments 1 and 2 are presented in the Appendix.

There was a practice list of 43 stimuli constructed for each experiment ( 37 were common across experiments). The practice lists primarily included exception words, so that participants received practice in the regularization task. The practice lists included $30 \mathrm{ex}-$ ception words, 8 regular words, and 5 nonwords. In addition, 4 buffer words ( 2 exception, 1 regular, and 1 nonword) were selected to serve at the beginning of the test block and were used in both experiments.

\section{Procedure}

At the beginning of each experimental session, the participants were seated comfortably in front of the computer while they were given instructions for the experiment. The participants in the normal naming task were told to name each word and nonword aloud as quickly and as accurately as possible. The participants in the regularization task were told to pronounce the words by applying the spelling-to-sound principles of the language as quickly as possible. To clarify the task, the participants were given examples of how to pronounce an irregular letter string (e.g., pronounce the word have so that it rhymes with gave). Each session began with the presentation of a practice block of trials, during which the experimenter provided feedback to the participants, which included examples of how to correctly regularize the words that they were unable to pronounce. This was intended mainly to help participants receiving the regularization instructions to learn the task.

On each trial during the experimental block, the following sequence of events occurred: (1) A warning tone was sounded; (2) a

Table 1

Characteristics of Stimuli

\begin{tabular}{crrrrr}
\hline Stimuli & Frequency & Length & Number of Friends & Number of Enemies & Coltheart's $N$ \\
\hline Regular words & & & & & \\
High frequency & 352 & 4.35 & 9.10 & 0.40 & 9.10 \\
Low frequency & 6 & 4.40 & 8.45 & 0.10 & 7.38 \\
Exception words & & & & & \\
High frequency & 524 & 4.35 & 1.35 & 4.33 & 4.91 \\
Low frequency & 10 & 4.47 & 2.15 & 4.73 & 5.40 \\
\hline
\end{tabular}


330-msec blank screen was presented; (3) five asterisks were displayed at the center of the screen for $330 \mathrm{msec}$; (4) a blank screen was again presented for $330 \mathrm{msec}$; (5) the target stimulus was presented until a response was detected, at which time the screen was blanked; (6) the experimenter entered a digit to score the trial, using the keyboard; and (7) the keypress initiated a 1,500-msec intertrial interval. Trials were scored as follows: (1) correct (clear pronunciation appropriate to the task instructions); (2) incorrect (clear pronunciation, but not appropriate to the task instructions; e.g., pronouncing pint normally when it should have been pronounced so that it rhymed with mint); and (3) invalid pronunciation (i.e., the participant produced a dysfluent pronunciation or the voice key was triggered by an extraneous sound).

\section{RESULTS}

The following procedure was used to eliminate extreme scores that might unduly influence estimates of the mean of a condition. First, for each participant, any response less than $200 \mathrm{msec}$ or greater than $2,500 \mathrm{msec}$ was removed from the analyses. Of the remaining observations, any response latency that deviated by 2.5 standard deviations from the participant's overall mean were also eliminated. The proportion of the observations that were considered outliers in Experiment 1 was .03 for the normal naming and .04 for the regularization task, whereas the corresponding proportions for Experiment 2 were .02 for the normal naming and .05 for the regularization task.

Before turning to the results, it is important to note that the same pattern of significant effects was obtained when separate analyses were conducted on each of the experiments. The omnibus design was a 2 (Experiment 1 vs. 2) $\times 2$ (normal naming vs. regularization task) $\times 2$ (regularity) $\times 2$ (frequency) mixed factor analysis of variance (ANOVA), with the dependent measures being response latency and proportion correct. We will report results both from analyses by participants $\left(F_{1}\right)$ and from analyses by items $\left(F_{2}\right)$. All the reported effects are significant at the .05 level, unless otherwise indicated. It is noteworthy that because the experiment factor did not interact with any of the factors below, we will not discuss it any further.

\section{Response Latencies}

The mean response latencies and proportion correct as a function of task (normal naming vs. regularization), regularity (regular vs. exception), and frequency (high vs. low) are presented in Table 2. One should first note that, as was expected, the regularization task produced relatively high error rates and slow response latencies, as compared with normal naming. More important, as is shown in Table 2, the effect of word frequency appears to be modulated by both task and regularity, as is reflected by a reliable task $\times$ frequency $\times$ regularity interaction $\left[F_{1}(1,98)=8.69, M S_{\mathrm{e}}=2,886.02 ; F_{2}(1,148)=4.33\right.$, $M S_{e}=4,975.401$. In order to further interpret this interaction, we conducted separate ANOVAs on the regular words and the exception words. For the regular words, there was a highly reliable task $\times$ frequency interaction $\left[F_{1}(1,98)=38.12, M S_{\mathrm{e}}=1,663.15 ; F_{2}(1,76)=16.35\right.$, $\left.M S_{\mathrm{e}}=4,135.68\right]$, indicating that high-frequency words were named more slowly than low-frequency words in the regularization task $[t(51)=4.04]$, whereas in the normal naming task, low-frequency words were named more slowly than high-frequency words $[t(49)=6.66]$. Turning to the exception words, although there were main effects of both task $\left[F_{1}(1,98)=171.93, M S_{\mathrm{e}}=68,668.40\right.$; $\left.F_{2}(1,72)=1,745.49, M S_{\mathrm{e}}=5,861.70\right]$ and frequency $\left[F_{1}(1,98)=10.81, M S_{\mathrm{e}}=3,118.57 ; F_{2}(1,72)=3.36\right.$, $\left.M S_{\mathrm{e}}=8,803.41, p=.07\right]$, there was no evidence of a frequency $\times$ task interaction (both $F \mathrm{~s}<1$ ).

\section{Proportion Correct}

As is shown in Table 2, the proportion correct data (eliminating both errors and outliers) followed a pattern similar to that for the response latency data. (Most of the errors in the regularization condition involved failures to regularize the exception words, which did not differ as a function of frequency - that is, $17 \%$ and $21 \%$, respectively, for high-and low-frequency words.) Specifically, there again appears to be some modulation of the word frequency effect by task and regularity. Although the overall interaction among task, frequency, and regularity did not reach significance $\left[F_{1}(1,98)=1.68, M S_{\mathrm{e}}=0.0037\right.$, $\left.p<.20 ; F_{2}(1,148)<1.00\right]$, separate ANOVAs on the proportion correct for the regular words produced a reliable task $\times$ frequency interaction $\left[F_{1}(1,98)=6.25, M S_{\mathrm{e}}=\right.$ $\left.0.003 ; F_{2}(1,76)=4.82, M S_{\mathrm{e}}=0.003\right]$, which reflected the fact that there was a small $(-2 \%)$ reversal of the frequency effect for regular words in the regularization task, but a normal (3\%) word frequency effect for normal naming of these same items. Again, the task $\times$ frequency

Table 2

Mean Response Latency (RT; in Milliseconds) and Proportion Correct (PC) as a Function of Frequency, Regularity, and Task

\begin{tabular}{|c|c|c|c|c|c|c|}
\hline \multirow[b]{2}{*}{ Task } & \multicolumn{2}{|c|}{ High Frequency } & \multicolumn{2}{|c|}{ Low Frequency } & \multicolumn{2}{|c|}{ Frequency Effect } \\
\hline & RT & $\mathrm{PC}$ & RT & $\mathrm{PC}$ & RT & $\mathrm{PC}$ \\
\hline \multicolumn{7}{|c|}{ Regular Words } \\
\hline Regularization & 976 & .87 & 933 & .89 & -43 & -.02 \\
\hline Normal naming & 533 & .99 & 559 & .96 & 26 & .03 \\
\hline \multicolumn{7}{|c|}{ Exception Words } \\
\hline Regularization & 1,024 & .69 & 1,047 & .62 & 23 & .07 \\
\hline Normal naming & 539 & .95 & 568 & .87 & 29 & .08 \\
\hline
\end{tabular}


interaction did not approach significance for the exception words (both $F \mathrm{~s}<1$ ). ${ }^{3}$

\section{Task by Lexicality Interaction}

In addition to the analyses on the word conditions, we also conducted a set of analyses to address changes in the lexicality effect across task instructions. If participants were attending more to sublexical processes in the regularization task and nonwords are completely dependent on sublexical processes, one might expect the effect for the nonwords in the regularization task to be equivalent to that in the normal naming task. As is shown in Table 3 , this clearly did not occur. Of course, it is possible that we did not find this main effect because this comparison was a between-subjects manipulation and participants may have set a higher temporal threshold for responding (owing to the higher error rate) in the regularization condition, as compared with the normal naming condition. In this light, it might be more informative to consider the lexicality $\times$ task interaction. Here, we focus on only the regular words versus the nonwords, because the exception words involved different responses. As is shown, there is clear evidence of an interaction in both response latencies $\left[F_{1}(1,98)=26.21, M S_{\mathrm{e}}=2,696\right.$; $\left.F_{2}(1,156)=28.14, M S_{\mathrm{e}}=4,076.79\right]$ and proportion correct $\left[F_{1}(1,98)=95.05, M S_{\mathrm{e}}=0.003 ; F_{2}(1,156)=97.90\right.$, $\left.M S_{\mathrm{e}}=0.006\right]$, which reflects the fact that there was virtually no difference between response latencies to words and nonwords under the regularization condition $(1 \mathrm{msec}$ and -.02 correct), whereas there was a large difference between words and nonwords under the normal naming condition ( $76 \mathrm{msec}$ and .14 correct). Thus, it appears that the direction of attention to nonlexical information in the regularization task eliminates the lexicality effect found in normal naming.

\section{GENERAL DISCUSSION}

The major results of the present experiments are clear: Specifically, for a set of regular words that produced a normal word frequency effect in speeded naming (i.e., high-frequency words being responded to slower than low-frequency words), the effect of word frequency was reversed under regularization instructions that directed the participants' attention to sublexical processes. This pattern was found in two experiments across different sets of items in response latency and, to some extent, in error rates. Moreover, because for regular words, the same re-

Table 3

Mean Response Latency (RT; in Milliseconds) and Proportion Correct (PC) as a Function of Lexicality and Task

\begin{tabular}{|c|c|c|c|c|c|c|}
\hline \multirow[b]{2}{*}{ Task } & \multicolumn{2}{|c|}{ Word } & \multicolumn{2}{|c|}{ Nonword } & \multicolumn{2}{|c|}{$\begin{array}{c}\text { Lexicality Effect } \\
\text { (Nonword - Word) }\end{array}$} \\
\hline & RT & $\mathrm{PC}$ & RT & $\mathrm{PC}$ & RT & PC \\
\hline Regularization & 954 & .88 & 955 & .90 & 1 & -.02 \\
\hline Normal naming & 546 & .98 & 622 & .84 & 76 & .14 \\
\hline
\end{tabular}

sponse was produced with both normal naming and regularization instructions, this pattern is not constrained by differences in the nature of the articulatory response. In contrast to the regular words, there was little change in the word frequency effect for the exception words under normal naming and regularization instructions.

What constraints do the present data provide regarding the lexical processing system? First, the present data indicate that participants cannot simply turn off the lexical processing route, via the regularization instructions, and totally rely on a frequency-independent sublexical processing route. If participants could focus on a frequencyindependent route in the regularization conditions, then (1) there should be no influence of word frequency, (2) the nonwords should at least be as fast in the regularization condition as in the normal naming condition, (3) there should be no influence of regularity (because this effect presumably is due to competition between the two routes), and (4) one should not find such slow response latencies in the regularization condition. Thus, if there indeed exists separate lexical and sublexical processing routes, the present results indicate that participants cannot easily eliminate the influence of the lexical route under the regularization instructions.

Although it is clearly the case that participants cannot simply focus on a frequency-independent sublexical route, it is also the case that the present results yielded a strong influence of the instructional manipulations on the effects of word frequency, regularity, and lexicality. Specifically, if the participants were relying on the same processing architecture in both the normal naming and the regularization conditions, one would expect similar frequency effects in both conditions. However, the present results produced clear interactions among task, regularity, and frequency, suggesting that a simple account in terms of differing the allocation of resources within the normal reading system will be insufficient to accommodate these results. In addition, the elimination of the large lexicality effect (found in normal naming) in the regularization task is consistent with the notion that there was at least partial control of lexical and nonlexical information.

Before turning to a potential theoretical account of the present results, one needs to be assured that the slow response latencies in the regularization conditions do not undermine the importance of the present findings. Of course, one might expect slow responses under this condition because the participants are required to use their lexical processing system in an unusual manner. This pattern is reminiscent of the Stroop task, in which colornaming latencies are slow relative to word-naming latencies. Clearly, considerable insight has been gained regarding attentional control and lexical processing via color-naming latencies in the Stroop task. ${ }^{4}$ Moreover, it is worth noting here that the difference between word naming and color naming increases as the number of colors in the response set increases (see, e.g., Kanne, Balota, Spieler, \& Faust, 1998). Given the relatively large 
set of responses in the regularization condition, it should be no surprise that we found such a large difference in response latency across the normal naming and the regularization conditions.

Although the regularization condition is much slower than the normal naming condition, we would argue that, like Stroop performance, one can still gain evidence about the processing system by requiring participants to select an alternative processing pathway. In fact, one might argue that there is more than simple surface level similarity to the interference effects found in the Stroop task. Specifically, if one assumes that the lexical pathway is more modulated by the whole-word frequency than is the sublexical pathway ${ }^{5}$ and that the regularization instructions indeed encouraged subjects to rely more heavily on the sublexical pathway, one might expect more interference from high-frequency words than from low-frequency words. The notion here is that the high-frequency words would produce more lexical competition because of their stronger pathway, as compared with low-frequency words (just as an incongruent word produces interference in color naming), thereby reversing the word frequency effect.

Unfortunately, however, this Stroop-like interpretation encounters difficulties. In particular, this interpretation cannot explain why the frequency effect is intact in the exception word condition. If the reversal of the frequency effect is due to the suppression of the lexical pathway, one would expect a similar reversal in the case of exception words. In this light, the reversal of the word frequency effect cannot simply be attributed to increased interference from the lexical processing pathway. Moreover, the relatively long response latencies and the threeway interaction between frequency, regularity, and task suggest an alternative interpretation that emphasizes the role of decision processes. One possibility is that readers of English have explicit knowledge of at least some spelling-to-sound rules, such as those taught in elementary school phonics curricula. The participants in the present experiments may have used this kind of metalinguistic/heuristic knowledge to generate responses in the regularization task. Because generating a correct response for the exception words requires producing an unfamiliar pronunciation for a familiar stimulus, a verification procedure may have been instituted in which the heuristically generated pronunciation was compared against the correct pronunciation. If the two pronunciations did not match, the participant could be reasonably certain that the heuristically generated pronunciation was a correct response. This strategy is plausible because a salient feature of the task is the requirement that participants produce something other than a lexically driven response - that is, the typical response for exception words. It may also provide an explanation for the frequency $x$ regularity $X$ task interaction. The frequency effect remains intact for exception words under this strategy because, in order to compare the heuri tically generated response with the correct response, the reading system still needs to generate the correct response. The lexically driven pronunciation is made available more slowly for the low-frequency exception words than for the highfrequency exception words.

We now turn to the most intriguing aspect of the datathat is, the reversal of the word frequency effect for regular words. This reversal may arise because the heuristic procedure and the reading system do not generate different responses but actually generate the same response for regular words. This agreement between the reading system and the heuristic procedure could be a source of interference - that is, this is not what the system is tuned to detect in the regularization task. The more familiar the word, the more likely it is that participants will "doublecheck" to ensure that they are generating the pronunciation by rule, and not by the normal reading pathway. Why might the participant do a double check when there is a high degree of coherence? Within a dynamical systems approach (e.g., Stone \& Van Orden, 1994), one might consider the regularization condition as a search for incoherence between the orthographic and the phonological systems. Because one would expect that highfrequency words would produce the strongest coherence signal-that is, the lexical and the orthographic-tophonological pathways would be very consistent-they actually induce a type of double check to ensure that the participant did not miss an alternative pronunciation. Of course, all of the remaining conditions would be less likely to cohere as strongly (i.e., low-frequency regular words and high- and low-frequency exception words).

Interestingly, a similar interference phenomenon has been revealed in the Stroop task. In particular, Heathcote, Popiel, and Mewhort (1991), Mewhort, Braun, and Heathcote (1992), and Spieler, Balota, and Faust (1996) found, via ex-Gaussian response time distribution analyses, that the congruent condition (in which both the word and the color pathways produce the same response) decreased the leading edge of the distribution (as reflected by the mu parameter), as compared with a neutral condition, but also produced more responses in the slow tail of the distribution (as reflected by the tau parameter). The similarity between the two tasks is this: In both cases, participants are asked to actively ignore one source of information (the correct pronunciation of the presented word) and focus on another (the color in Stroop, the regularized pronunciation here). When these two pieces of information agree, most of the responses actually show a benefit, but a cost is incurred on some trials. In the regularization task, the probability that this cost will be incurred is positively correlated with the frequency of the word in question, because the greater familiarity of the regular pronunciations of these words makes them more anomalous in the context of the experiment. Assuming that the slowdown for high-frequency regular words arises from a shift in the tail of the distribution also allows this account to handle the finding that regular words were faster overall in the regularization condition than were exception words. That is, the bulk of the responses benefited from the congruency of the heuristically generated 
and correct pronunciations, but a subset of the responses suffered from the double-check process in this condition.

At a more general level, we have suggested that the present results provide evidence of the role of attentional control in processing lexical information (also see Zevin $\&$ Balota, 2000). It seems quite important in developing models of word recognition to consider the flexibility of the processing system in accentuating and attenuating different processing pathways. Balota et al. (1999) have recently argued that, depending on the goals of the task, participants will accentuate or attenuate different processing pathways. In support of this argument, they point out that lexical decision performance may place a premium on familiarity/meaning information to discriminate words from nonwords, whereas speeded naming performance may place a relatively greater premium on spelling-to-sound correspondence to maximize the speed of the correct pronunciation of the orthographic string. The influence of attentional control systems nicely accommodates the fact that, in lexical decision, one typically finds larger frequency and meaning effects than in naming, whereas in naming performance, there are larger regularity effects than in lexical decision performance (see Balota et al., 1999, for a review of this literature).

In conclusion, the present results indicate that the word frequency effect can actually be reversed under conditions in which participants are instructed to generate rule-based pronunciations for exception words. These results have been viewed as providing converging evidence for the importance of considering the goals of the task and the pathways that participants engage to achieve those goals. In fact, coupled with the recent results of Balota and Spieler (1999), it is clear that one can find any pattern of frequency effect, depending on the task demands. Specifically, Balota and Spieler found large frequency effects $(63 \mathrm{msec})$ in the lexical decision task, smaller frequency effects $(21 \mathrm{msec})$ in the naming task, and no frequency effect $(4 \mathrm{msec})$ in a rhyme decision task for the same set of words. Now, we can add to this mix the present reversals of the word frequency effect of $43 \mathrm{msec}$. Thus, one can find the full range of frequency effects, depending on the task demands. In this way, it should be clear that it is necessary to go beyond the notion of a static lexical processing system and consider the flexibility of the processing system and the pathways that are attenuated and accentuated by attentional control systems to accomplish the goals of a task.

\section{REFERENCES}

ANDREws, S. (1982). Phonological recoding: Is the regularity effect consistent? Memory \& Cognition, 10, 565-575.

Balota, D. A., \& Ferraro, F. R. (1996). A dissociation of frequency and regularity effects in pronunciation performance across young adults, older adults and individuals with senile dementia of the Alzheimer type. Journal of Memory \& Language, 32, 573-592

Balota, D. A., \& SpIEleR, D. H. (1999). Word-frequency, repetition, and lexicality effects in word recognition tasks: Beyond measures of central tendency. Journal of Experimental Psychology: General, 128, $32-55$.
Balota, D. A., PaUl, S. T., \& SPIELER, D. H. (1999). Attentional control of lexical processing pathways during word recognition and reading. In S. Garrod \& M. Pickering (Eds.), Language processing (pp. 15-57). London: Psychology Press.

BALUCH, B., \& Besner, D. (1991). Visual word recognition: Evidence for strategic control of lexical and nonlexical routines in oral reading. Journal of Experimental Psychology: Learning, Memory, \& Cognition, 17, 644-652.

ColthearT, M. (1978). Lexical access in simple reading tasks. In G. Underwood (Ed.), Strategies in information processing (pp. 151-216). San Diego: Academic Press.

Coltheart, M., Curtis, B., Atkins, P., \& Haller, M. (1993). Models of reading aloud: Dual-route and parallel distributed processing approaches. Psychological Review, 100, 589-608.

Coltheart, M., Davelaar, E., Jonasson, J., \& Besner, D. (1977). Access to the internal lexicon. In S. Dornic (Ed.), Attention and performance VI (pp. 535-555). Hillsdale, NJ: Eribaum.

FoDOR, J. A. (1983). The modularity of the mind. Cambridge, MA: MIT Press.

FredERIKSEN, J. R., \& KROLL, J. F. (1976). Spelling and sound: Approaches to the internal lexicon. Journal of Experimental Psychology: Human Perception \& Performance, 2, 361-379.

Frost, R., Katz, L., \& Bentin, S. (1987). Strategies for visual word recognition and orthographical depth: A multilingual comparison. Journal of Experimental Psychology: Human Perception \& Performance, 13, 104-115.

Heathcote, A., Popiel, S. J., \& Mewhort, D. J. K. (1991). Analysis of response time distributions: An example using the Stroop task. Psychological Bulletin, 109, 340-347.

JARED, D. (1997). Evidence that strategy effects in word recognition reflect changes in output timing rather than changes in processing route. Journal of Experimental Psychology: Learning, Memory, \& Cognition, 23, 1424-1438.

KanNe, S. M., Balota, D. A., Spieler, D. H., \& Faust, M. E. (1998). Explorations of Cohen, Dunbar, and McClelland's (1990) connectionist model of Stroop performance. Psychological Review, 105, 174-187.

KUČERA, H., \& FranCIS, W. (1967). Computational analysis of presentday American English. Providence, RI: Brown University Press.

LukATELA, G., \& TuRVEY, M. T. (1994). Visual lexical access is initially phonological: 2 . Evidence from phonological priming by homophones and pseudohomophones. Journal of Experimental Psychology: General, 123, 331-353.

LUPKER, S. J., Brown, P., \& Colombo, L. (1997). Strategic control in a naming task: Changing routes or changing deadlines? Journal of Experimental Psychology: Learning, Memory, \& Cognition, 23, 570-590.

Marshall, J. C., \& Newcombe, F. (1980). The conceptual status of deep dyslexia: An historical perspective. In M. Coltheart, K. Patterson, \& J. C. Marshall (Eds.), Deep dyslexia (pp. 1-21). London: Routledge \& Kegan Paul

McClelland, J. L., \& RumelharT, D. E. (1981). An interactive activation model of context effects in letter perception: Pt. 1. An account of basic findings. Psychological Review, 88, 375-407.

Mewhort, D. J. K., Braun, J. G., \& Heathcote, A. (1992). Response time distributions and the Stroop task: A test of the Cohen, Dunbar, and McClelland (1990) model. Journal of Experimental Psychology: Human Perception \& Performance, 18, 872-882.

MIDGLEY-WEST, L. (1979). Phonological encoding and subject strategies in skilled reading. Unpublished doctoral thesis, University of London, Birbeck College.

Monsell. S., Patterson, K. E., Graham, A., Hughes, C. H., \& MilROY, R. (1992). Lexical and sublexical translation of spelling to sound: Strategic anticipation of lexical status. Journal of Experimental Psychology: Learning, Memory, \& Cognition, 18, 452-467.

Monsell, S., Patterson, K. E., Tallon, J., \& Hill, J. (1989, January). Voluntary surface dyslexia: A new argument for two processes in oral reading? Paper presented at a meeting of the Experimental Psychology Society, London.

MORTON, J. (1969). The interaction of information in word recognition. Psychological Review, 76, 165-178.

NEELY, J. H. (1977). Semantic priming and retrieval from lexical memory: 
Roles of inhibitionless spreading activation and limited capacity attention. Journal of Experimental Psychology: General, 106, 226-254.

PAAP, K. R., \& NOEL, R. W. (1991). Dual route models of print to sound: Still a good horse race. Psychological Research, 53, 13-24.

Plaut, D. C., McClelland, J. L., Seidenberg, M. S., \& PatterSON, K. (1996). Understanding normal and impaired word reading: Computational principles in quasi-regular domains. Psychological Review, 103, 56-115.

Seidenberg, M. S., \& McClelland, J. L. (1989). A distributed, developmental model of word recognition and naming. Psychological Review, 96, 523-568.

Seidenberg, M. S., Waters, G. S., Barnes, M. A., \& Tanenhaus, M. K. (1984). When does irregular spelling or pronunciation influence word recognition? Journal of Verbal Learning \& Verbal Behavior, 23, 383-404

Shallice, M. S., \& Warrington, E. K. (1980). Single and multiple component central dyslexic syndromes. In M. Coltheart, K. Patterson, \& J. C. Marshall (Eds.), Deep dyslexia (pp. 119-145). London: Routledge \& Kegan Paul.

Simpson, G. B., \& KaNG, H. (1994). The flexible use of phonological information in word recognition in Korean. Journal of Memory \& Language, 33, 319-331.

SPIEler, D. H., Balota, D. A., \& Faust, M. E. (1996). Stroop performance in healthy younger and older adults and in individuals with dementia of the Alzheimer's type. Journal of Experimental Psychology: Human Perception \& Performance, 22, 461-479.

StONE, G. O., \& VAN ORden, G. C. (1994). Building a resonance framework for word recognition using design and system principles. Journal of Experimental Psychology: Human Perception \& Performance, 20, 1248-1268.

STROOP, J. R. (1935). Studies of interference in serial verbal reactions. Journal of Experimental Psychology, 18, 643-661.

SWINNEY, D. A. (1979). Lexical access during sentence comprehension: (Re)consideration of context effects. Journal of Verbal Learning \& Verbal Behavior, 18, 645-659.

TABossi, P., \& LAGHI, L. (1992). Semantic priming in the pronunciation of words in two writing systems: Italian and English. Memory \& Cognition, 20, 303-313.
Taraban, R., \& McClelland, J. L. (1987). Conspiracy effects in word recognition. Joumal of Memory \& Language, 26, 608-631.

Zevin, J. D., \& Balota, D. A. (2000). Priming and attentional control of lexical and sublexical pathways during naming. Journal of Experimental Psychology: Learning, Memory, \& Cognition, 26, 121-135.

\section{NOTES}

1. It should be noted here that Monsell, Patterson, Tallon, and Hill (1989) have also reported, at a conference, results from a regularization task. However, the details of this study are currently unavailable.

2. Although the present study focuses on speeded naming of English words, it should be noted that studies of other languages with varying levels of spelling-to-sound consistencies have provided somewhat clearer evidence regarding the control of processing pathways (e.g., Baluch \& Besner, 1991; Simpson \& Kang, 1994; Tabossi \& Laghi, 1992).

3 . It is worth noting that the typical frequency $\times$ regularity interaction that is found in the normal naming task did not occur in the present response latency data (both $F \mathrm{~s}<1$ ). However, this interaction was reliable in the proportion correct data $\left[F_{\mathrm{I}}(1,48)=16.35, M S_{\mathrm{e}}=0.002\right.$; $\left.F_{2}(1,148)=3.70, M S_{\text {e }}=0.008, p<.06\right]$.

4. One might argue that there is an important distinction between the present results and the Stroop task. Specifically, in the Stroop task, it is the theoretically irrelevant stimulus component (color) that becomes task relevant. In the regularization condition, one might argue that it is the theoretically relevant component (spelling-to-sound correspondence) that becomes more task relevant. We acknowledge that there is this distinction. However, in both cases, it is the theoretically relevant component (normal word reading) that actually needs to be controlled to access a different type of information (color or spelling-to-sound correspondence).

5. It is important to emphasize here that we would not argue that the sublexical pathway is insensitive to frequency information. However, in this case, the frequency information is most likely carried by units smaller than the whole word (e.g., bigrams, trigrams, and onsets and rhymes). Because we attempted to equate the neighborhood characteristics across the high- and low-frequency words, there should have been relatively little influence of such sublexical frequency-dependent information in the regularization condition. 
APPENDIX

Critical Stimuli and Matched Nonwords in Experiments 1 and 2

\begin{tabular}{|c|c|c|c|c|c|}
\hline \multicolumn{4}{|c|}{ Stimuli } & \multirow{3}{*}{\multicolumn{2}{|c|}{ Nonwords }} \\
\hline \multicolumn{2}{|c|}{ Regular Words } & \multicolumn{2}{|c|}{ Exception Words } & & \\
\hline High Frequency & Low Frequency & High Frequency & Low Frequency & & \\
\hline \multicolumn{6}{|c|}{ Experiment 1} \\
\hline beach & apple & answer & bold & bealt & answal \\
\hline least & fogy & front & facade & martel & frolp \\
\hline miss & pill & once & sword & sem & sape \\
\hline still & stink & touch & bury & sumpel & waty \\
\hline came & blast & come & famine & blasp & cobe \\
\hline list & glass & gross & morale & milt & grold \\
\hline more & pomp & said & wand & sib & senade \\
\hline these & summit & two & choir & trafe & whon \\
\hline dark & bolo & done & folk & cade & dode \\
\hline main & hack & most & pour & moke & oceal \\
\hline name & $\operatorname{sip}$ & senate & wily & sopt & steab \\
\hline time & wade & want & chute & waim & woft \\
\hline faster & chomp & earth & $\operatorname{gin}$ & darp & earsh \\
\hline market & morass & move & remind & pamtle & onal \\
\hline power & sobs & steak & wolf & stimp & toult \\
\hline train & weed & whom & glove & weeb & worb \\
\hline hall & chump & father & soot & mank & fasher \\
\hline member & pamper & ocean & worm & pilk & pode \\
\hline see & sock & sure & & stist & twy \\
\hline west & yelp & word & & yeld & yuke \\
\hline \multicolumn{6}{|c|}{ Experiment 2} \\
\hline back & bail & been & bold & bealt & answal \\
\hline firm & dock & give & gin & blasp & cobe \\
\hline heat & heel & said & scald & cade & dode \\
\hline side & scrape & touch & wash & darp & earsh \\
\hline boat & bait & come & bush & mank & fasher \\
\hline first & drip & great & glove & martel & frolp \\
\hline hope & kite & show & soot & milt & grold \\
\hline sort & seep & two & wolf & moke & oceal \\
\hline dark & bump & done & choir & pamtle & onal \\
\hline flat & fern & mind & gross & pilk & pode \\
\hline keep & $\operatorname{mink}$ & some & swab & sem & sape \\
\hline spend & slurp & want & worm & sib & senade \\
\hline date & crate & find & chute & sopt & steab \\
\hline gain & gale & most & plaid & stimp & toult \\
\hline plant & perk & steak & sword & stist & twy \\
\hline spot & wane & whom & folk & sumple & waty \\
\hline deep & dame & front & roll & trafe & whon \\
\hline game & girth & move & wand & waim & woft \\
\hline rest & rift & sure & & weem & worb \\
\hline wish & yank & word & & yeld & yuk \\
\hline
\end{tabular}

(Manuscript received August 3, 1998;

revision accepted for publication January 18, 2000.) 